Article

\title{
Aphasia after acute ischemic stroke in Romania: epidemiology and impact on tertiary care resources
}

\author{
Adina Stan ${ }^{1,2,3}$, Stefan Strilciuc* ${ }^{* 1,3}$, Nicoleta Gherghel ${ }^{3}$, Angela Cozma ${ }^{4}$, Cristian Alexander ${ }^{3}$, Silvina Ilut ${ }^{1,2,3}$, \\ Alina Blesneag ${ }^{1,2,3}$, Vitalie Vacaras ${ }^{1,2,3}$, Delia Stanca ${ }^{1,2,3}$, Horatiu Stan ${ }^{1,3}$, Dafin Muresanu ${ }^{1,2,3}$

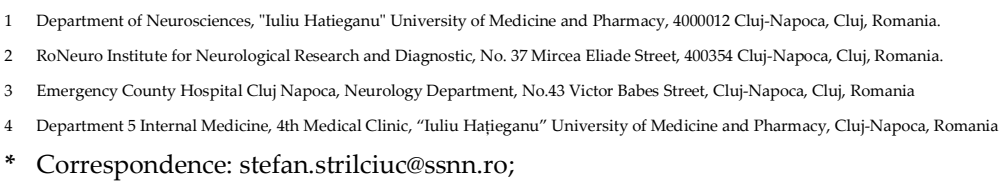

\begin{abstract}
We aimed to reveal the disease burden of aphasia after acute ischemic stroke (AIS) at the national level and investigate the impact of aphasia on tertiary care resources and patient outcomes. The local database from the Cluj-Napoca Emergency County Hospital (CNECH), the second largest stroke center in Romania was used to export demographics, baseline clinical and laboratory data, inpatient length of stay (LOS), NIH Stroke Scale (NIHSS), and discharge modified Rankin Scale (mRS) score data for all AIS patients admitted during March 2019. Of 92 patients included in the study, 30 (32.6 \%) had aphasia on admission. In a marginally significant unadjusted hierarchical multiple regression model, individuals with aphasia had a LOS of 1.86 days longer than stroke survivors without aphasia. In an adjusted version of the model, the NIHSS score at baseline was a significant predictor for LOS. In addition, the presence of aphasia was associated with a 1.49 increase in the mean mRS score. Aphasia was a marginally significant predictor for increased LOS. Presence of aphasia was more likely to produce a poor functional outcome. Considering an estimated impact of approximately EUR 3 million on direct medical expenditure annually, future policymaking efforts should improve prevention of stroke and improved access to post-stroke aphasia care in Romania.
\end{abstract}

Keywords: aphasia, acute ischemic stroke, length of stay, mRS, NIHSS

\section{Introduction}

In the context of ever-growing pressure on health system resources related to population ageing, increase in morbidity and mortality due to non-communicable diseases, as well as the impact of the COVID-19 pandemic, culprits such as stroke, which have significant contributions to the global burden of disease have come into the spotlight of health economists seeking to find ways to increase value for money.

Data from 32 European countries showed that in 2017 the cost of stroke reached $€ 60$ billion, with direct medical costs accounting for almost half of this staggering figure. Health expenditure for stroke varies greatly between western and eastern European countries [1]. Romania has one of the lowest proportions of Gross Domestic Product allocated to healthcare, standing historically at around half of the European Union average. As evidenced by the most recent cost of illness studies, access to proper stroke care infrastructure, staff, and supplies are limited accordingly.

Aphasia, or difficulty in producing or comprehending spoken or written language, is one of the most debilitating manifestations of AIS, affecting $21 \%-38 \%$ of all cases [2]. 
Patients with post-stroke aphasia are shown to have increased mortality, long-term disability, and more complications than stroke survivors without aphasia [3]. Despite anecdotal reports that access to care such as speech therapy for aphasia is notoriously low in Romania, few studies have reported insight on the epidemiology of aphasia at the national level and its impact on hospital resources. Moreover, data regarding the current cost and precise cost structure related to stroke are scarce [4]. We hypothesize that inappropriate access to care for post-stroke aphasia may lead to substantial direct and indirect costs from regardless of economic perspective.

This pilot project aimed to reveal the disease burden of aphasia after AIS and investigate its impact on tertiary care resources and functional patient outcomes.

\section{Materials and Methods}

\subsection{Study population}

A retrospective analysis of data regarding all patients with AIS admitted to a primary stroke centre from CNECH during May 2019 was performed. Data extracted from our local registry included demographic variables, baseline clinical and laboratory variables, LOS, NIHSS, discharge mRS scores and discharge location. Only patients with index symptomatic AIS in the MCA territory and a pre-stroke mRS score of 0 or 1 were included.

\subsection{Exposure and outcome definitions}

The NIHSS was performed for each patient on admission [5]. Aphasia was defined as having a score of 1 or greater on admission NIHSS question 9. Aphasia type was classified according to Boston Classification System [6]. Outcomes of interest included LOS and $\mathrm{mRS}$ score at discharge. Poor functional outcome was defined as a mRS score of 3 or above at discharge [7].

\subsection{Statistical analysis}

Frequencies, means, and standard deviations were used for the descriptive analysis. Two hierarchical multiple regression analyses were conducted with LOS and mRS scores as dependent variables. In the first step, an unadjusted model was tested, with aphasia as a single predictor. Age and NIHHS scores were added as predictors in the second step to adjust for their effects. Assumptions of the multiple regression analysis were also tested to ensure the reliability of the analysis. Analyses were run in SPSS (IBM, Armonk, NY, USA).

\section{Results}

\subsection{Assumptions' check}

Collinearity analysis showed no multicollinearity in the data, as proved by VIF scores below ten and tolerance scores above 0.2. VIF values were as follows: unadjusted models: $\mathrm{VIF}=1$, tolerance $=1$; adjusted models: VIF scores of 1.34, 1.07, 1.39; tolerance scores of $0.74,0.93,0.71$. Analysis of Durbin-Watson statistic for the model with LOS was 2.23 , for the model with mRS score was 2.22 , therefore showing that the values of the residuals are independent. The standardized residuals versus standardized predicted values plot indicated no signs of funnelling, suggesting that the homoscedasticity assumption is met. The P-P plot suggests that the assumption of normality of the residuals was met-inspection of the Cook's distance values no compelling cases (all values lower than 1). 


\subsection{Main analysis}

The descriptive statistic of all variables is shown in Tables 1 and 2. Separate values are provided for patients with and without aphasia. Hierarchical regression analysis in the model with LOS as dependent variable indicated in the unadjusted model that aphasia was a marginally significant predictor $(F(1,90)=3.79, p=.055)$, accounting for $0.040 \%$ of the variance in LOS.

Table 1. Baseline demographic information for patients with and without aphasia.

\begin{tabular}{lll}
\hline & Aphasia (N=30) & No apahsia (N= 62) \\
\cline { 2 - 3 } Variable & Frequency (percent) & Frequency (percent) \\
\hline Female sex & $20(66.7)$ & $30(48.4)$ \\
Thrombolysis & $7(23.3)$ & $7(11.3)$ \\
Medical history & & \\
Atrial fibrillation & $15(50)$ & $11(17.7)$ \\
Symptomatic Carotid stenosis & $1(3.3)$ & $6(9.7)$ \\
Smoking & $5(16.7)$ & $12(19.4)$ \\
Dyslipidemia & $24(80)$ & $52(83.9)$ \\
Hypertension & $24(80)$ & $52(83.9)$ \\
Aphasia type & & \\
Broca & $9(30)$ & \\
Mixed transcortical & $12(40)$ & $2(3.2)$ \\
Global & $7(23.3)$ & \\
Anomic & $2(6.7)$ & $57(91.9)$ \\
Discharge deposition & $2(6.7)$ & \\
Home & $20(66.7)$ & \\
Another department/center & $4(13.3)$ & \\
Deceased & $4(13.3)$ & \\
Senior care centre & & \\
\hline
\end{tabular}

Table 2. Demographic and outcome information for patients with and without aphasia.

\begin{tabular}{lll} 
& Aphasia $(\mathbf{N}=\mathbf{3 0})$ & No aphasia $(\mathbf{N}=\mathbf{6 2})$ \\
\hline Variable & Mean (Standard Deviation) & Mean (Standard Deviation) \\
& Minimum - Maximum & Minimum - Maximum \\
\hline Age (years) & $74.86(11.51)$ & $70.83(10.82)$ \\
& $41-97$ & $40-88$ \\
LOS (days) & $9.76(5.51)$ & $7.90(3.58)$ \\
& $0-25$ & $0-19$
\end{tabular}




$\begin{array}{lll}\text { NIHSS score } & 13.70(8.74) & 5.80(4.94) \\ & 1-30 & 1-25 \\ \text { Discharge } & 3.26(2.03) & 1.77(1.62) \\ \text { mRS score } & 0-6 & 0-6\end{array}$

As shown by regression coefficients in Table 3 - unadjusted model, having aphasia marginally increased the mean LOS by 1.86 days. Adjusting for the effects of age and NIHSS score resulted in an overall significant model $(F(3,88)=4.53, p=.005)$ and explained an additional $0.134 \%$ of the variance in LOS. Examination of individual predictors indicated NIHSS score to be the only significant predictor in the model. Effects of aphasia and age were not significant. As shown by regression coefficients in Table 3 - adjusted model, the predicted LOS for a patient without aphasia, of mean age and mean NIHSS score was 11.30 days. A one unit increase in the NIHSS score predicted a 0.20 day increase of this mean LOS.

Hierarchical regression analysis in the model with mRS score as dependent variable indicated in the unadjusted model that aphasia was a significant predictor $(\mathrm{F}(1,90)=$ $14.43, \mathrm{p}=<.001$ ), accounting for $0.138 \%$ of the variance in $\mathrm{mRS}$ scores. As shown by regression coefficients in Table 4 - unadjusted model, having aphasia increased the mean mRS score by 1.49 points compared to the mean scores of 1.77 for patients without aphasia. Adjusting for the effects of age and NIHSS score resulted in an overall significant model $(\mathrm{F}(3,88)=41.68, \mathrm{p}=<.001)$ and explained an additional $0.587 \%$ of the variance in $\mathrm{mRS}$ scores. Examination of individual predictors indicated significant effects for age and NIHSS score. As shown by regression coefficients in Table 4 - adjusted model, the predicted mRS score for a patient without aphasia, of mean age and mean NIHSS score was -1.26. A one unit increase in the NIHSS score predicted a 0.18 point increase of this mean mRS score.

Table 3. Regression coefficients for length of stay (LOS) model.

Unadjusted model

\begin{tabular}{lllllll}
\hline \multicolumn{1}{c}{ B } & SE & $\beta$ & $t$ & $\mathrm{P}$ \\
Constant & 7.90 & 0.547 & & 14.46 & $<.001$ \\
Aphasia & 1.86 & 0.957 & 0.201 & 1.94 & .055 \\
Adjusted model & & & & \\
\hline & $\mathrm{B}$ & $\mathrm{SE}$ & $\beta$ & $t$ & $\mathrm{P}$ \\
\hline Constant & 11.301 & 2.847 & & 3.97 & $<.001$ \\
Aphasia & 0.537 & 1.065 & 0.058 & 0.50 & .615 \\
Age & -0.064 & 0.040 & -0.165 & -1.60 & .113 \\
NIHHS & 0.201 & 0.069 & 0.340 & 2.90 & .005 \\
\hline
\end{tabular}


Table 4. Regression coefficients for modified Rankin Scale (mRS) model.

$\begin{array}{cccccc}\text { Unadjusted model } & & & & \\ & \text { B } & \text { SE } & \beta & t & \mathrm{p} \\ \text { Constant } & 1.774 & 0.224 & & 7.91 & <.001 \\ \text { Aphasia } & 1.492 & 0.393 & 0.372 & 3.80 & <.001\end{array}$

\begin{tabular}{lllllll}
\multicolumn{1}{c}{ Adjusted model } & & & & \\
\hline & B & SE & $\beta$ & $t$ & $\mathrm{p}$ \\
Constant & -1.260 & 0.851 & & -1.48 & .143 \\
Aphasia & -0.066 & 0.319 & -0.017 & -0.208 & .836 \\
Age & 0.028 & 0.012 & 0.164 & 2.31 & .023 \\
NIHHS & 0.183 & 0.021 & 0.716 & 8.85 & $<.001$ \\
\hline
\end{tabular}

\section{Discussion}

This study set out to evaluate burden, access, and the impact of post-stroke aphasia on tertiary care resources and functional patient outcomes in the Romanian patient pathway. Our main results show that aphasia is associated with prolonged LOS during acute stroke admission and a higher mRS score at discharge. These data are in accordance with recent studies highlighting the underrecognized effect of communication impairment on stroke patients' outcomes [2,3]. Over the last few years, studies have managed to demonstrate that aphasic patients have more disability at discharge (defined as an mRS score of 3-6) than non-aphasics [8]. These findings remain even after accounting for NIHSS scores and inpatient complications. In contrast, patients with hemiparesis are not at higher odds of having a discharge mRS of 3-6 after adjusting for the same parameters [2]. Furthermore, aphasia at baseline was also associated with a poorer $\mathrm{mRS}$ at three months in a retrospective analysis on 8904 stroke patients [9]. These data align with our results that show that aphasia increases the mean mRS score at discharge by 1.49 points.

In the present study, aphasic and non-aphasic patients had a mean LOS of 9.76 and 7.9 days, respectively, lower than the previously reported mean for other countries [11] but similar to that obtained in another Romanian regional study [4]. We found that the presence of aphasia marginally prolongs LOS by 1.86 days, as compared to non-aphasic patients. However, when controlling for age and NIHSS score, the effect of aphasia alone was no longer significant. This observation does not necessarily imply that aphasia no longer has an effect, as the NIHSS score includes a measure of aphasia, which we could not separate from the total score due to limitations in data collection. In a retrospective analysis on 1847 stroke patients, after adjusting for NIHSS score and inpatient 
complication, a 1.22 day increase in LOS was reported in aphasics [2]. In addition, in a retrospectively examined cohort of 3200 stroke patients, the LOS was 8.6 days in aphasic patients compared to 7.2 days in non-aphasic ones [3].

Several possible explanations are accounting for a longer LOS in these patients. One is that a failure to communicate and understand commands can impair physical therapy, thus prolonging motor recovery $[10,12]$. Moreover, aphasics have been shown to develop more complications (such as sepsis, pneumonia, neurological worsening, and myocardial infarction) than non-aphasics [2]. This finding could result from difficulty expressing complaints and symptoms worsening or properly following instructions [2]. Another pertinent explanation is that these patients sometimes have difficulties finding a suitable rehabilitation placement either because they do not necessarily have associated motor deficits or because they cannot perform occupational therapies $[3,10]$.

Considering that acute LOS is responsible for more than $65 \%$ of the total inpatient costs, prediction of this outcome variable is essential for the precise planning of the health care system resources. The LOS-associated costs account for acute hospital beds, intensive use of neuroimaging, medication, and paramedical resources [13]. Unfortunately, data regarding the costs of stroke care in Romania are limited. However, a recent analysis showed that the average cost per stroke inpatient care episode summed up to EUR 995 in 2017, while the mean LOS was 9.9 days [14]. Thus, we estimated that the average cost for a one-day hospital stay for stroke was about EUR 100 in our country in 2017. Assuming a $30 \%$ aphasia rate among 55.000 new ischemic stroke cases annually [15] and considering a 1.86 day LOS prolongation, we concluded that aphasia adds EUR 3 million annually to the care of these patients in Romania.

Compared to a recent study that estimated that aphasia adds a cost of about EUR 1.77 billion annually in the US [2], the financial burden seems to be minimal in our country. In truth, with an annual expenditure of only EUR 7.15 per capita and a total allocation of EUR 140 million in 2017 for stroke, Romania was at the bottom among European countries and far away from the American expenditure for stroke [14]. Consequently, aphasia's financial burden is considerable and represents a challenge for the limited local healthcare system budget.

We acknowledge several limitations to this study, including a small patient sample and a larger group of non-aphasics as compared to aphasics. This could account for aphasia not being a predictor for longer LOS in the adjusted model instead of similar research [2]. Second, only the total NIHSS score was available for analysis, so we could not evaluate which individual NIHSS sub-components apart from aphasia correlate with LOS.

The strengths of the present study lie in the novelty of the data, being the first in Romania to address the financial burden of stroke-related aphasia. Future research could focus on how different types of aphasia impact acute stroke outcomes. In addition, upcoming studies could investigate whether those outcomes are influenced by receiving aphasia therapy during hospitalization.

\section{Conclusions}

Overall, our study showed that aphasia in stroke patients is associated with increased disability at discharge and prolonged LOS, which significantly add to the burden of the health care system in Romania. These findings suggest that better management of communication disorders during acute stroke hospitalization is needed. Likewise, the results of this study can be used as a baseline for measuring cost-effectiveness of new or improved stroke care strategies. 


\section{Supplementary Materials: N/A.}

Author Contributions: Conceptualization, A.S., S.S., H.S, D.M.; validation, A.S., S.S., N.G., A.C., C.A, S.I., A.B., V.V., D.S., H.S, D.M.; formal analysis, A.S., N.G., A.C., C.A; investigation, A.S., S.I., A.B., V.V., D.S., D.M.; data curation, S.S., H.S; writing-original draft preparation, A.S. N.G., A.C., C.A,; writing-review and editing, A.S., S.S., N.G., A.C., C.A, S.I., A.B., V.V., D.S., H.S, D.M.; supervision, A.S., S.S., V.V., H.S, D.M; project administration, A.S. . All authors have read and agreed to the published version of the manuscript.

Funding: This research received no external funding.

Institutional Review Board Statement: Ethical review and approval were waived for this study, due to the anonymized and aggregated nature of patient data used for a retrospective analysis.

Informed Consent Statement: Not applicable.

Conflicts of Interest: The authors declare no conflict of interest.

\section{References}

1. Luengo-Fernandez R, Violato M, Candio P, Leal J. Economic burden of stroke across Europe: A population-based cost analysis. Eur Stroke J. 2020 Mar;5(1):17-25.

2. Boehme AK, Martin-Schild S, Marshall RS, Lazar RM. Effect of Aphasia on acute stroke outcomes. Neurology. 2016 Nov 29;87(22):2348-2354.

3. Ellis C, Simpson AN, Bonilha H, Mauldin PD, Simpson KN. The one-year attributable cost of poststroke Aphasia. Stroke. 2012 May;43(5):1429-31.

4. Strilciuc S, Grad DA, Mixich V, Stan A, Buzoianu AD, Vladescu C, Vintan MA. Societal Cost of Ischemic Stroke in Romania: Results from a Retrospective County-Level Study. Brain Sci. 2021 May 24;11(6):689.

5. Brott T, Adams HP Jr, Olinger CP, Marler JR, Barsan WG, Biller J, Spilker J, Holleran R, Eberle R, Hertzberg V, et al. Measurements of acute cerebral infarction: a clinical examination scale. Stroke. 1989 Jul;20(7):864-70.

6. A. Kertesz, Assessment of Aphasia, in: T. Incagnoli, G. Goldstein, C.J. Golden (Eds.), Clinical Application of Neuropsychological Test Batteries, Springer US, Boston, MA, 1986: pp. 329-360.

7. Banks JL, Marotta CA. Outcomes validity and reliability of the modified Rankin scale: implications for stroke clinical trials: a literature review and synthesis. Stroke. 2007 Mar;38(3):1091-6.

8. Flowers HL, Silver FL, Fang J, Rochon E, Martino R. The incidence, co-occurrence, and predictors of dysphagia, dysarthria, and Aphasia after first-ever acute ischemic stroke. J Commun Disord. 2013 May-Jun;46(3):238-48.

9. Ali M, Lyden P, Brady M; VISTA Collaboration. Aphasia and Dysarthria in Acute Stroke: Recovery and Functional Outcome. Int J Stroke. 2015 Apr;10(3):400-406.

10. Dickey L, Kagan A, Lindsay MP, Fang J, Rowland A, Black S. Incidence and profile of inpatient stroke-induced Aphasia in Ontario, Canada. Arch Phys Med Rehabil. 2010 Feb;91(2):196-202

11. Appelros P. Prediction of length of stay for stroke patients. Acta Neurol Scand. 2007 Jul;116(1):15-9.

12. Galski T, Bruno RL, Zorowitz R, Walker J. Predicting length of stay, functional outcome, and aftercare in the rehabilitation of stroke patients. The dominant role of higher-order cognition. Stroke. 1993 Dec;24(12):1794-800

13. Ng YS, Tan KH, Chen C, Senolos GC, Chew E, Koh GC. Predictors of Acute, Rehabilitation and Total Length of Stay in Acute Stroke: A Prospective Cohort Study. Ann Acad Med Singap. 2016 Sep;45(9):394-403.

14. Lorenzovici L, Székely A, Csanádi M, Gaál P. Cost Assessment of Inpatient Care Episodes of Stroke in Romania. Front Public Health. 2020 Dec 4;8:605919.

15. Uivarosan D, Bungau S, Tit DM, Moisa C, Fratila O, Rus M, Bratu OG, Diaconu CC, Pantis C. Financial Burden of Stroke Reflected in a Pilot Center for the Implementation of Thrombolysis. Medicina (Kaunas). 2020 Jan 28;56(2):54. 